\title{
Preparation and properties of polylactic acid-based composite fluorescent membrane based on layer-by-layer assembly
}

\author{
M. M. Wang, H. L. Li, M. R. Liu* \\ State Key Laboratory of Pulp and Paper Engineering, South China University of Technology, 510640 Guangzhou, China
}

Received 14 December 2018; accepted in revised form 5 March 2019

\begin{abstract}
In this paper, a kind of multi-functional polylactic acid-based membrane with adjustable aggregate thickness and properties was prepared. When the number of assembled layers was 20 bilayers, the mean thickness of every bilayer was found to be $10.75 \pm 0.25 \mathrm{~nm}$, and the tensile strength was increased by $20 \%$ compared with the polylactic acid (PLA) films. The assembled layered double hydroxides (LDHs) could serve as a type of promising organic-inorganic luminescent material, and endow PLA with photoluminescent properties. Besides, the photoluminescence of the composite films was discovered to be highly tunable by simply adjusting the structural constituents of LDHs, and the fluorescence intensity was uniformly enhanced as the number of assembled layers increases, which was not eliminated by the isolation due to cellulose nanocrystals (CNCs). Moreover, the composite films exhibited excellent fluorescence stability in comparison with the literature. After being repeatedly bleached 1000 times by confocal laser scanning fluorescence microscope, the fluorescence intensity of the PLA/(CNCs/Mg$\mathrm{Al}-\mathrm{Eu} \mathrm{LDHs})_{10}$ film remained $96 \%$. It could be concluded that the immobilization of CNCs and LDHs realized the microscopic controllability of the optical functional molecular spacing. With satisfactory fluorescence, the resulting PLA-based membranes exhibited considerable application potential in optoelectronic devices and anti-counterfeiting packaging.
\end{abstract}

Keywords: polymer composites, polylactic acid, layer-by-layer assembly, photoluminescence, controllability

\section{Introduction}

Polylactic acid (PLA), a kind of polymer material from the synthesis of natural materials used in packaging, biomedicine and other fields, with good degradability, biocompatibility as well as similar mechanical properties to general purpose plastics, is still limited by its high hydrophobicity, low brittleness and poor thermal stability in practical applications. In consequence, scientists carried out a series of modification to enhance its properties, such as copolymerization modification, blend modification and composite modification [1-3]. Compared with those methods, the unique advantage of layer-by-layer self-assembly technique (LBL) lied in material diversity and superior controllability of the assembly process [4]. More specifically, a wide variety of materials incorporating inorganic or organic polycations, polyanions and nanoparticles could be applied to the performance of the LBL deposition. In the polymer area, LBL was an appropriate approach for generating novel nanocomposite structures with certain compositions or morphology. For instance, cellulose nanocrystals (CNCs) and chitosan were assembled onto PLA to build a dense, well-packed and well-adsorbed multilayer biocoating to improve the water vapor barrier properties. Besides, the thickness increase was found to be uniform and controllable, with average thickness of one bilayer of around $1.6 \mathrm{~nm}$. The resulting heterogeneous biocoating possessed a tunable function because of the structural 
diversity and combined functionality, which was challenging to achieve through direct mixing or blending difference polymers [5]. As a sort of renewable and sustainable nanomaterials, typically produced by strong sulfuric acid hydrolysis of lignocellulosic biomass, CNCs could self-assemble in aqueous and other suspensions at a critical concentration, or under evaporation, into the chiral nematic organization to exhibit anisotropic structural color. Moreover, owing to the exceptionally high mechanical strength and chemical tunability, much research has been conducted considering the effect of using $\mathrm{CNCs}$ as the reinforcing component of different composites [6-9].

Layered materials are attractive targets for their rich fundamental physics and potential applications in multifunctional devices. For example, transition metal-bearing layered double hydroxides (LDHs) and montmorillonite were assembled by LBL to form a novel luminescent multiple ordered thin film, with ultra-prolonged lifetime [10]. Additionally, LDHs nanosheets were also successfully applied to the assembly of photoemissive thin-film which can suppress the $p-p$ stacking of chromophores, and thus made organic photofunctional molecules show excellent optical properties [11-13]. In addition, it was confirmed that the luminescence behavior of rare earth contained inorganic-organic hybrids, which had potential application for light-emitting materials [14, 15]. In comparison with conventional photo-functional polymer sensors and optoelectronic materials which were prone to entanglement and accumulation between molecules and then caused luminescence quenching, LDHs possessed higher luminous efficiency. On account of the flexibility composed of both metal hydroxide layers and interlayer anions, LDHs could be delaminated to make composite functional materials and widely used in separation, catalysis, electrochemistry, and optical functional materials [16-19].

Due to the presence of sulfate groups and polar hydroxyl groups in the cellulose nanocrystals' structure, the CNCs were negatively charged in aqueous media, while LDHs were a kind of hydrotalcite-like clay with positive charged layer. Considering the likelihood of empowering special physicochemical characteristics by the synthesis of organic-inorganic hybrid materials based on polymers, herein both $\mathrm{CNCs}$ and LDHs were assembled onto the aminolyzed PLA. The target of this investigation was to prepare a biodegradable photoluminescent polymer with adjustable fluorescence intensity, without using organic dye or quantum dots. The resulting composite films were expected to exhibit better mechanical properties on basis of the reinforcing properties of nanocellulose. In addition, the introduction of LDHs nanosheets was aimed at endowing the modified PLA with the photoluminescent properties.

\section{Experimental section}

\subsection{Materials}

The bleached eucalyptus pulp ( $\alpha$-cellulose $\geq 90 \%$ ) was supplied by Yueyang Paper Co. Ltd., (Hunan, China). PLA (PLLA, $M_{\mathrm{n}}=50000, M_{\mathrm{w}}=100000$ ) films $(d=40 \pm 0.5 \mu \mathrm{m})$ were supplied by Jinjia Plastics Co. Ltd., (Dongguan, China). 1,6-hexanediamine, propanol, $\mathrm{Mg}\left(\mathrm{NO}_{3}\right)_{2} \cdot 6 \mathrm{H}_{2} \mathrm{O}, \mathrm{Al}\left(\mathrm{NO}_{3}\right)_{3} \cdot 9 \mathrm{H}_{2} \mathrm{O}$, $\mathrm{Eu}\left(\mathrm{NO}_{3}\right)_{3} \cdot 6 \mathrm{H}_{2} \mathrm{O}, \mathrm{NaNO}_{3}, \mathrm{NaOH}$, sulfuric acid, hydrochloric acid and ninhydrin were all purchased from Guangzhou Chemical Reagent Factory. All reagents were analytical reagents and used without further purification. Deionized water was utilized during the experiments.

\subsection{Processing of PLA}

The PLA membranes were immersed in the alcohol/water solution for 2-3 hours to remove the oily dirt, and then rinsed and dried. Then, the membranes were immersed in the distilled 1,6-hexanediamine/ propanol solution with a concentration of $0.06 \mathrm{~g} \cdot \mathrm{ml}^{-1}$ at $58^{\circ} \mathrm{C}$ for $3 \mathrm{~min}$, which were subsequently rinsed and dried to obtain the aminolyzed PLA membranes with a thickness of $\sim 40 \mu \mathrm{m}$. Finally, the aminolyzed PLA membranes were dipped in the $0.012 \mathrm{~mol} \cdot \mathrm{l}^{-1}$ $\mathrm{HCl}$ solution and washed to ensure the charged state of PLA [20].

\subsection{Preparation of cellulose nanocrystals}

The suspension of CNCs was prepared on the basis of sulfuric acid hydrolysis method [21, 22]. Briefly, the wood pulp was oven-dried and milled to pass a 40-mesh sieve. Subsequently, $5 \mathrm{~g}$ wood pulp powder (to oven dry) was added into $43 \mathrm{ml} 64 \mathrm{wt} \%$ sulfuric acid under mechanical stirring. The hydrolysis was conducted at $45^{\circ} \mathrm{C}$ for $50 \mathrm{~min}$, and then terminated by quenching with 10 -fold cold water. The suspension was washed using two repeated centrifuge cycles. Afterward, the supernatant was dialyzed against water for several days until the dialysate became 
neutral. The obtained CNCs suspension was then sonicated in an ice bath to disrupt the large $\mathrm{CNCs}$ aggregates.

\subsection{Preparation and exfoliation of LDHs}

$\mathrm{Mg}-\mathrm{Al}$-Eu LDHs were prepared using coprecipitation method [23]. $60 \mathrm{mmol} \mathrm{Mg}\left(\mathrm{NO}_{3}\right)_{2} \cdot 6 \mathrm{H}_{2} \mathrm{O}$, $27 \mathrm{mmol} \quad \mathrm{Al}\left(\mathrm{NO}_{3}\right)_{3} \cdot 9 \mathrm{H}_{2} \mathrm{O}$ and $3 \mathrm{mmol}$ $\mathrm{Eu}\left(\mathrm{NO}_{3}\right)_{3} \cdot 6 \mathrm{H}_{2} \mathrm{O}$ were dissolved in $50 \mathrm{ml}$ water under vigorous stirring conditions, which were blended into a mixed salt solution. The mixed base solution was prepared by dissolving $160 \mathrm{mmol} \mathrm{NaOH}$ and $40 \mathrm{mmol} \mathrm{NaNO}_{3}$ in $80 \mathrm{ml}$ deionized water. Then, the base solution was dropwise added into the mixed salt solution in 2 hours under vigorous stirring and purging conditions with $\mathrm{N}_{2}$. The $\mathrm{pH}$ value of the solution was adjusted to around 10 with $1 \mathrm{~mol} \cdot \mathrm{l}^{-1} \mathrm{NaOH}$ solution and $1 \mathrm{~mol} \cdot \mathrm{l}^{-1} \mathrm{HCl}$ solution. The resulting mixture of the aqueous solution was heated for crystallization at $65^{\circ} \mathrm{C}$ for $24 \mathrm{~h}$ through centrifugation and washing, and then the product was put in a vacuum and oven dried at $50^{\circ} \mathrm{C}$. $0.2 \mathrm{~g} \mathrm{Mg}$-Al-Eu LDHs prepared above was mixed with $100 \mathrm{ml}$ formamide, and shaken vigorously at room temperature for 2 days to yield a transparent colloidal suspension consisting of exfoliated LDHs nanosheets. Then, it was centrifuged at $5000 \mathrm{rpm}$ for $10 \mathrm{~min}$ to remove the unexfoliated LDHs nanoparticles.

$\mathrm{Mg}-\mathrm{Al} \mathrm{LDHs}$ were prepared using the identical method. $60 \mathrm{mmol} \mathrm{Mg}\left(\mathrm{NO}_{3}\right)_{2} \cdot 6 \mathrm{H}_{2} \mathrm{O}$ and $30 \mathrm{mmol}$ $\mathrm{Al}\left(\mathrm{NO}_{3}\right)_{3} \cdot 9 \mathrm{H}_{2} \mathrm{O}$ were dissolved in $50 \mathrm{ml}$ water to make the mixed salt solution. The mixed base solution was prepared by dissolving $160 \mathrm{mmol} \mathrm{NaOH}$ and $40 \mathrm{mmol} \mathrm{NaNO}_{3}$ in $80 \mathrm{ml}$ deionized water. Then, it was synthesized, crystallized and exfoliated using the same way.

\subsection{Layer-by-layer assembly of multilayer films}

As shown in Figure 1, the PLA/(CNCs/LDHs $)_{n}$ films were fabricated in line with the following steps: (a) dip the aminolyzed PLA membrane into an aqueous solution of CNCs for $15 \mathrm{~min}$, and then rinse with deionized water and dry it under $\mathrm{N}_{2}$ flow; (b) dip the membrane attained above into exfoliated LDHs suspension for $15 \mathrm{~min}$, and then thoroughly rinse and dry it under $\mathrm{N}_{2}$ flow. A series of deposition operations were repeated $n$ times to produce composite multilayer films of PLA/(LDHs/CNCs $)_{\mathrm{n}}$.

\subsection{Characterization}

Scanning electron microscopy (SEM, Zeiss Merlin, Germany) at acceleration voltage of $5 \mathrm{kV}$ and transmission electron microscopy (TEM, H7650, Japan) with accelerating voltage of $300 \mathrm{kV}$ were used to observe the morphology, the aggregation state and the particle size of the CNCs and LDHs. ImageJ was applied to measure the morphology of CNCs. The molar mass of aminolyzed PLA membranes was measured by gel permeation chromatography (GPC, Waters 2695 , Singapore). The water contact angle of aminolyzed PLA membranes was measured by the contact
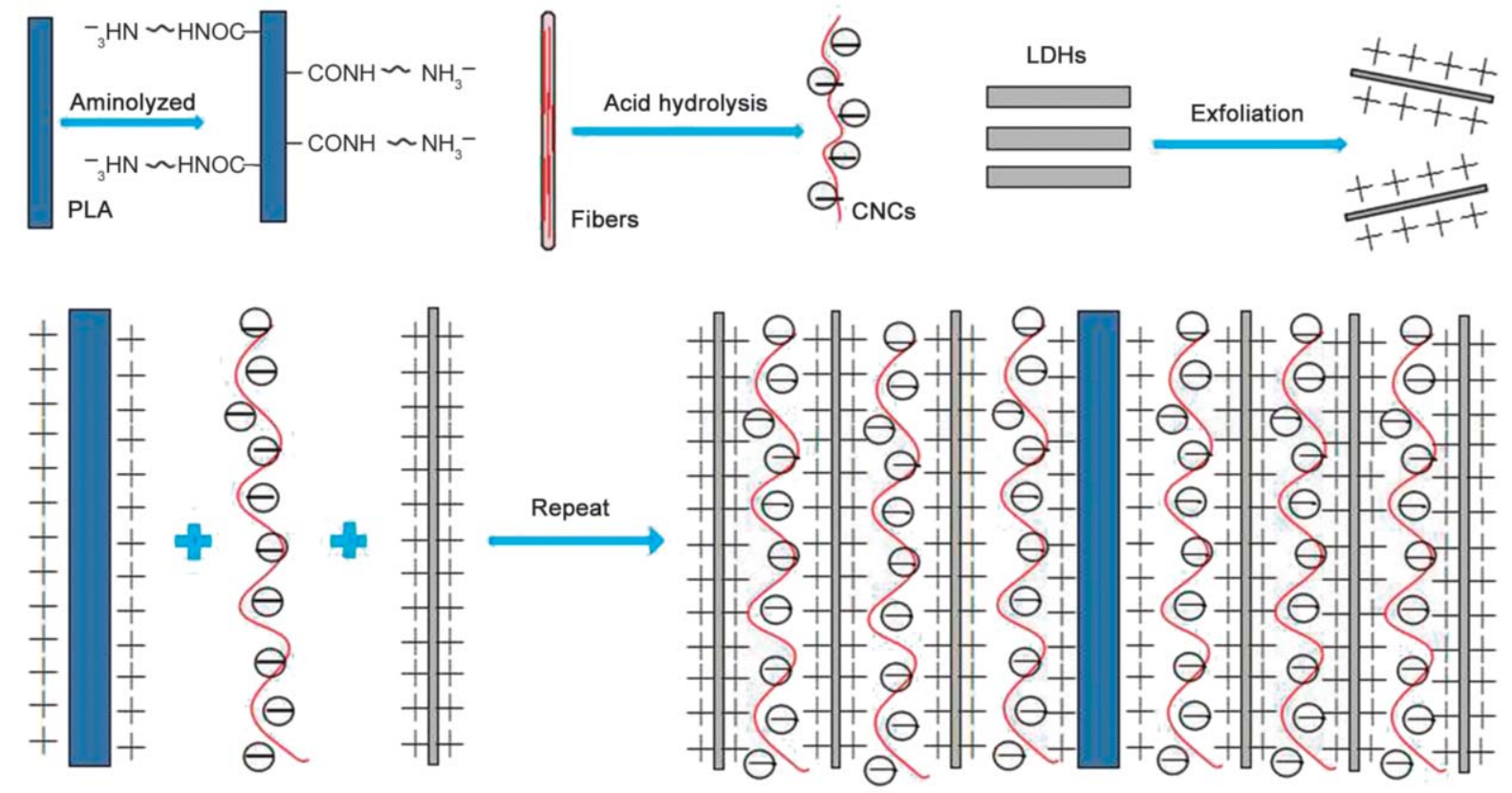

Figure 1. Schematic representation for the fabrication of PLA/(CNCs/LDHs) $n$ ultrathin films. 
angle tester (OCA40 MICRO, Data physics, America). Zeta potential of the CNCs suspension and LDHs nanosheets suspension was measured by a laser scattering analyzer (LA-960S, Horiba, Japan). The crystalline structure of LDHs was performed in a range of 5 to $80^{\circ}$ on X-ray diffraction (XRD, Bruker D8 Advance, Germany) at output power of $3 \mathrm{~kW}$ with $\mathrm{Cu}$ radiation. Fourier transform infrared spectroscopy of Mg-Al-Eu LDHs was determined with a spectrophotometer using the attenuated total reflection spectrum (FTIR-ATR, Bruker VerTex 70, Germany), ranged from 4000 to $400 \mathrm{~cm}^{-1}$. The ultraviolet-visible (UV-vis) spectrum in the range of 200 to $800 \mathrm{~nm}$ was recorded via a Shimadzu UV-2501PC spectrophotometer equipped with an integrating sphere detection system. The tensile mechanical properties of the composites were measured using a universal material testing machine (Instron 5565, Waltham, MA, USA) with $100 \mathrm{~N}$ load cells. The tested samples were cut into a $50 \mathrm{~mm} \times 10 \mathrm{~mm}$ rectangular shape, and the samples were stretched at a speed of $10 \mathrm{~mm} \cdot \mathrm{min}^{-1}$. Tensile properties reported here represent mean values of at least six samples' results. The fluorescence properties were characterized by the confocal laser scanning fluorescence microscope (CLSM, Leica TCS-SP5, Germany) with the $40 \times$ objective, and the photoluminescence emission spectrum (PLES, Fluoromax-4, Horiba, Japan) was measured by the fluorescence spectrophotometer under $395 \mathrm{~nm}$ direct excitation with a $150 \mathrm{~V}$ xenon lamp as the excitation source. Additionally, the bleaching properties of composites were characterized with the

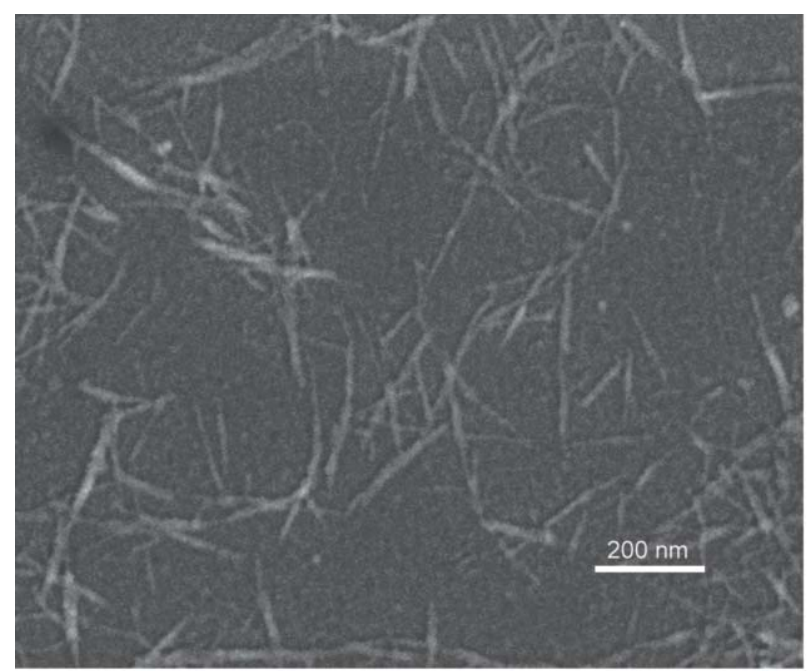

a)
FRAP-Mode of CLSM at the scan speed of $1400 \mathrm{~Hz}$ and the format of $256 \times 256$.

\section{Results and discussion}

\subsection{Characterization of aminolyzed PLA}

The aminolyzed PLA membrane was measured by ninhydrin method. Free $\mathrm{NH}_{3}{ }^{+}$and ninhydrin could produce blue reaction, and aminolyzed PLA could produce this blue reaction with ninhydrin. Meanwhile, the water contact angle of the aminolyzed PLA membrane was reduced from 88.6 to 66.8 , which also confirmed the reaction. After the aminolysis of the PLA, the molar mass was 53056, and the zeta potential value was nearly $+43.1 \mathrm{mV}$. It testified that positive charges were introduced onto the hydrophobic and neutral PLLA surface after aminolyzed in hexane diamine solution, and the PLA obtained free amino groups that were positively charged at neutral $\mathrm{pH}$, so that the electronic assembly can be processed.

\subsection{Morphology and structure of CNCs and Mg-Al-Eu LDHs nanosheets}

The morphology of the CNCs was observed by SEM and TEM, the length and the diameter were also statistically measured. As shown in Figure 2, CNCs had a relatively short rod shape with a length of $200 \pm 50 \mathrm{~nm}$ and a width of $15 \pm 5 \mathrm{~nm}$. Sulfuric acid hydrolysis of fibers resulted in the conversion of partial hydroxyl groups to sulfates, which were electronegative in aqueous media. The zeta potential value of synthesized CNCs was about $-33.1 \mathrm{mV}$ that was close to the reported value [24], which signified that

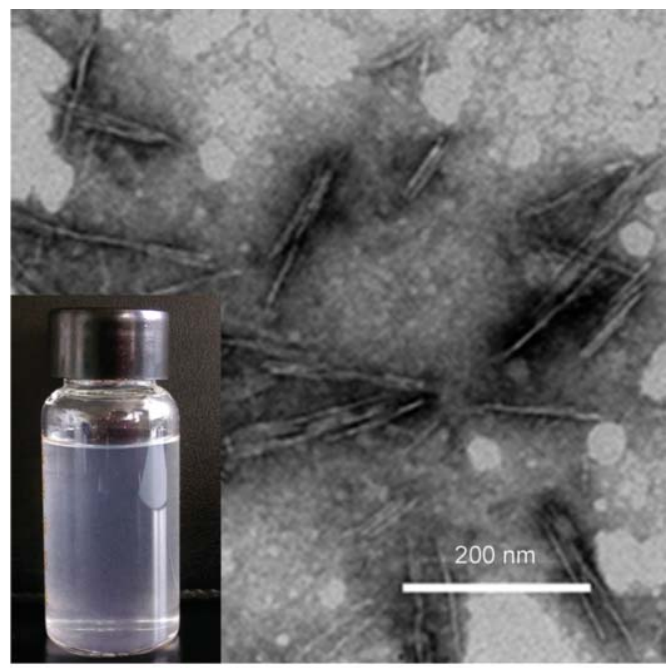

b)

Figure 2. SEM (a), TEM (b) image and optical photograph of CNCs. (a) SEM image of CNCs, (b) TEM image and optical photograph of CNCs 
the CNCs could be used to assemble nanoparticles carrying positive surface charges [25]. It was reported that multi-component hybrid thin films were prepared in the light of the hydrogen bonds and electrostatic interactions between sulfate ester groups and $\mathrm{NH}_{3}{ }^{+}$groups [26, 27].

LDHs samples were prepared by a coprecipitation method. The morphology of as-prepared Mg-Al-Eu LDHs was characterized by SEM. As presented in Figure 3a, the morphology of nanosheet samples was aggregated into clustering, and numerous thin nanosheet structures grew uniformly and formed the hierarchical flower-like architecture [28]. Additionally, the FT-IR spectrum of Mg-Al-Eu LDHs was given in Figure $3 \mathrm{~b}$. The peak at $3450 \mathrm{~cm}^{-1}$ as well as $1650 \mathrm{~cm}^{-1}$ was ascribed to the stretching modes of the surface hydroxyl group and the bending mode of interlayer water molecules, respectively. The peak at $430 \mathrm{~cm}^{-1}$ represented the stretching vibration absorption peak of M-O in the Mg-Al-Eu LDHs skeleton structure. In addition, the band attributed to $\mathrm{NO}_{3}{ }^{-}$

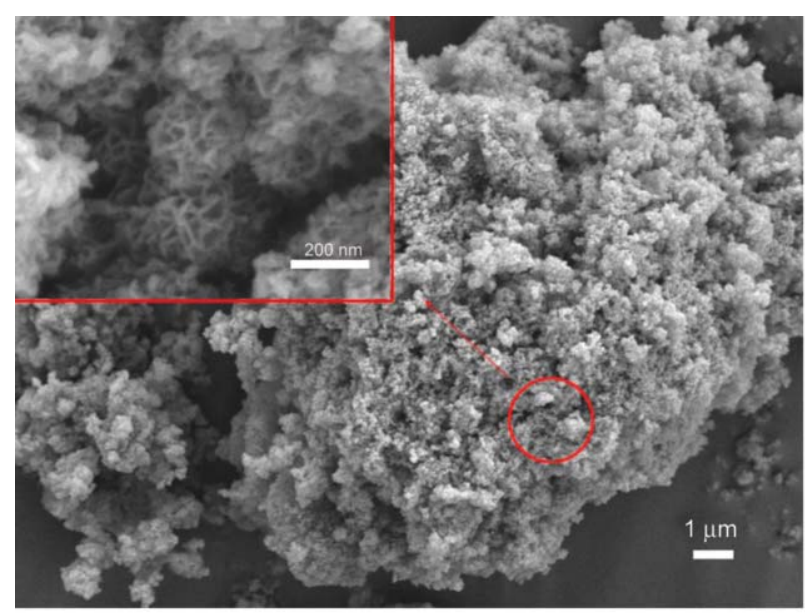

a)

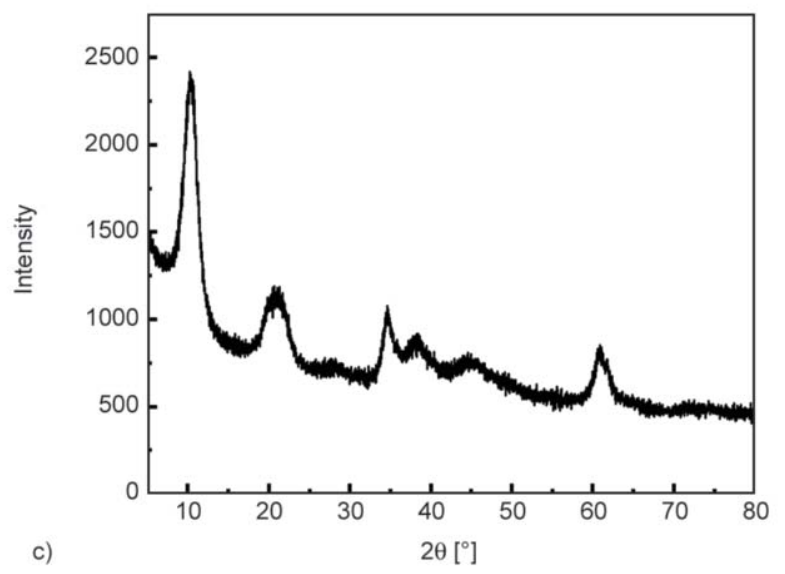

anions existed in $1380 \mathrm{~cm}^{-1}$, meaning that nitrate-type LDHs with superior delamination were prepared.

Furthermore, the crystal structure and phase composition of LDHs were detected by XRD. As indicated in Figure 3c, Mg-Al-Eu LDHs exhibited the diffraction peaks at $3.34,6.57,35.06$, and $61.03^{\circ}$, which corresponded to the (003), (006), (012), and (110) planes of a typical layered double hydroxide. And there was a proportional relationship between the layer spacings corresponding to the (003) and (006), indicating that the as-prepared $\mathrm{Mg}$-Al-Eu LDHs had a favorable layered structure [29]. The photoluminescence emission spectrum (PLES) was reflected in Figure $3 \mathrm{~d}$, and all the ${ }^{5} \mathrm{D}_{0} \rightarrow{ }^{7} \mathrm{~F}_{\mathrm{J}}(J=0,1,2,3,4)$ emission line characteristic of $\mathrm{Eu}^{3+}$ at 537, 590, 613, 648 and $699 \mathrm{~nm}$ were observed at room temperature. It demonstrated that the rare earth ion $\mathrm{Eu}^{3+}$ still retained the characteristic line after entering into the Mg-Al LDHs laminate [30].

After exfoliation of Mg-Al-Eu LDHs in formamide, transparent colloidal suspension was obtained, and

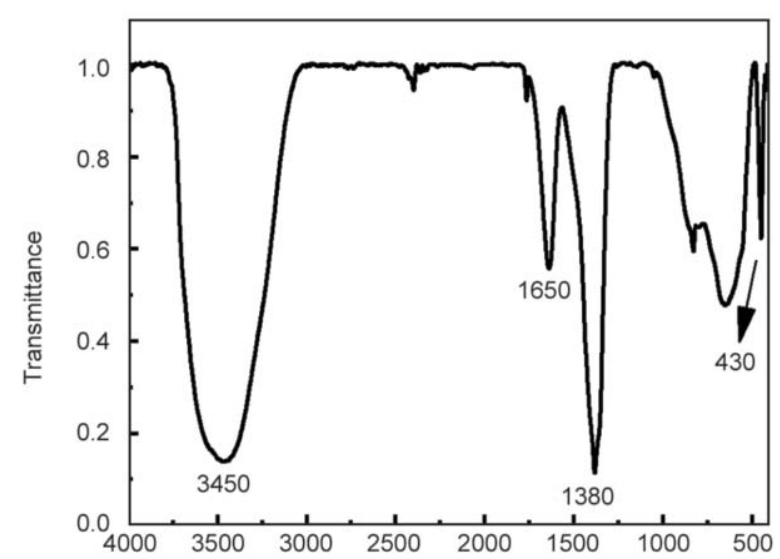

b)

Wave number $\left[\mathrm{cm}^{-1}\right]$

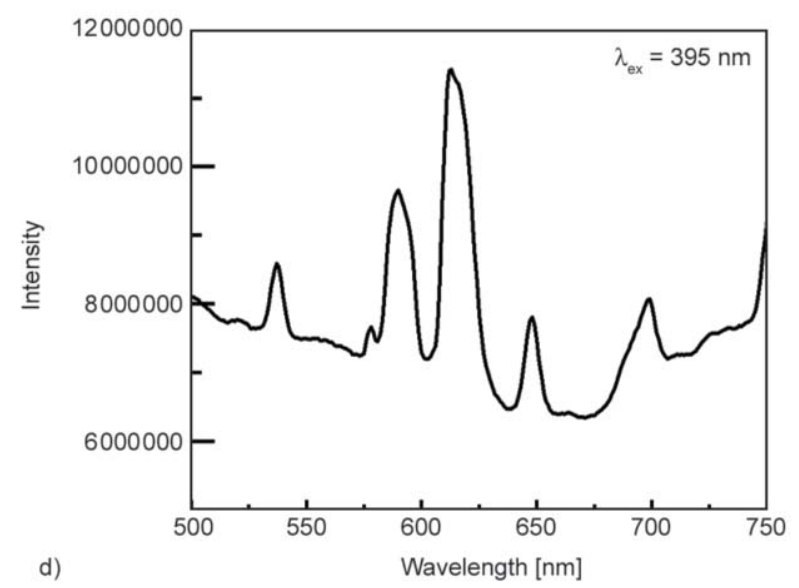

Figure 3. SEM image (a) and FTIR (b), XRD (c) and PLES (d) of Mg-Al-Eu LDHs. 


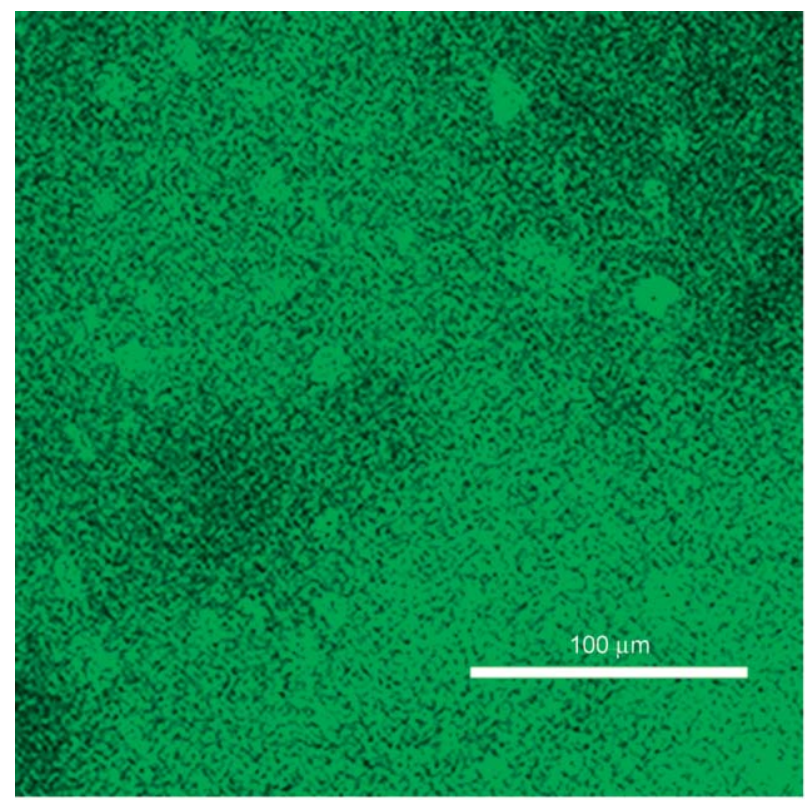

a)

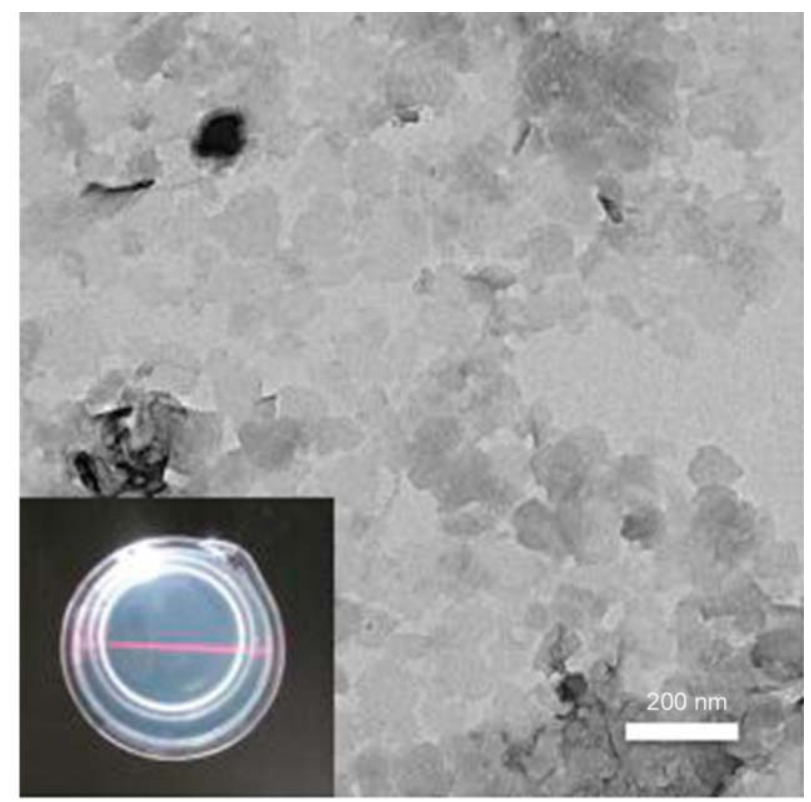

b)

Figure 4. CLSM image under 514 nm (a), TEM (b) image and optical photograph of Mg-Al-Eu LDHs nanosheet.

the properties of the unilamellar Mg-Al-Eu LDHs nanosheets were also characterized. As illustrated in Figure 4, well-dispersed plate-like Mg-Al-Eu LDHs nanosheets which fluoresced at $514 \mathrm{~nm}$ were observed by CLSM, with a uniform size about 60 $120 \mathrm{~nm}$ that was nearly equivalent to the size of the unstripped $\mathrm{Mg}$-Al-Eu LDHs precursor. Furthermore, the zeta potential value of the Mg-Al-Eu LDHs nanosheet was about $40 \mathrm{mV}$, implying that the obtained Mg-Al-Eu LDHs suspension could be used for LBL assembly and construction of nanodevices incorporating with other negative charged nanoparticles [31]. Hence, the CNCs and Mg-Al-Eu LDHs nanosheets could be assembled onto aminolyzed PLA, owing to the hydroxyl and sulfate groups on the surface of

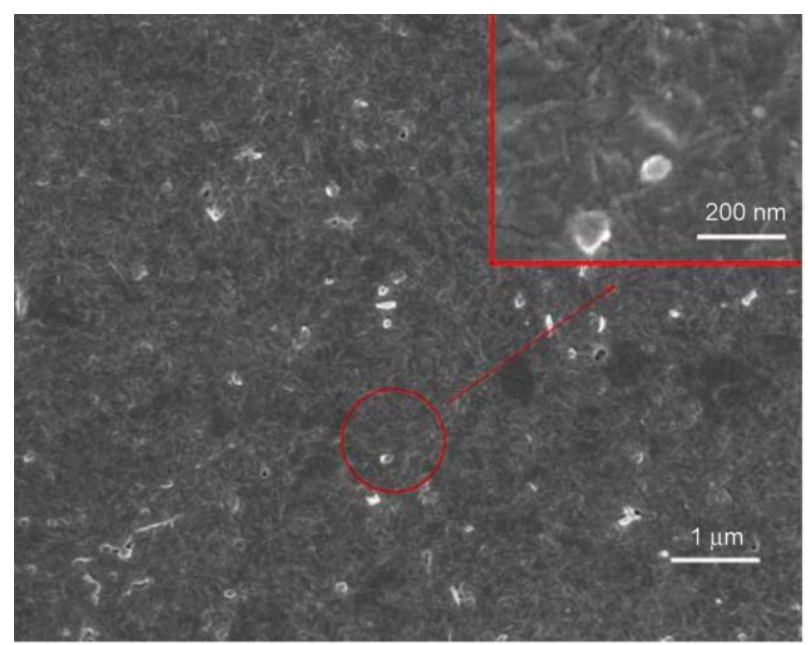

a)
$\mathrm{CNCs}$ and hydroxyl groups on that of $\mathrm{Mg}-\mathrm{Al}-\mathrm{Eu}$ LDHs nanosheets.

\subsection{Characterizations of composite membrane}

\subsubsection{Increasing rules of composite membrane}

The microstructure of PLA/(CNCs/Mg-Al-Eu LDHs $)_{n}$ composite films was characterized by SEM in Figure 5a, where CNCs and Mg-Al-Eu LDHs were densely stacked on the PLA surface, and the physiognomy of PLA/(CNCs/Mg-Al-Eu LDHs $)_{10}$ was continuous, uniform and flat. Moreover, the formation of network structure was readily observed in the insert picture of Figure 5a, which was favorable for the contact and the interfacial adhesion between the polymer

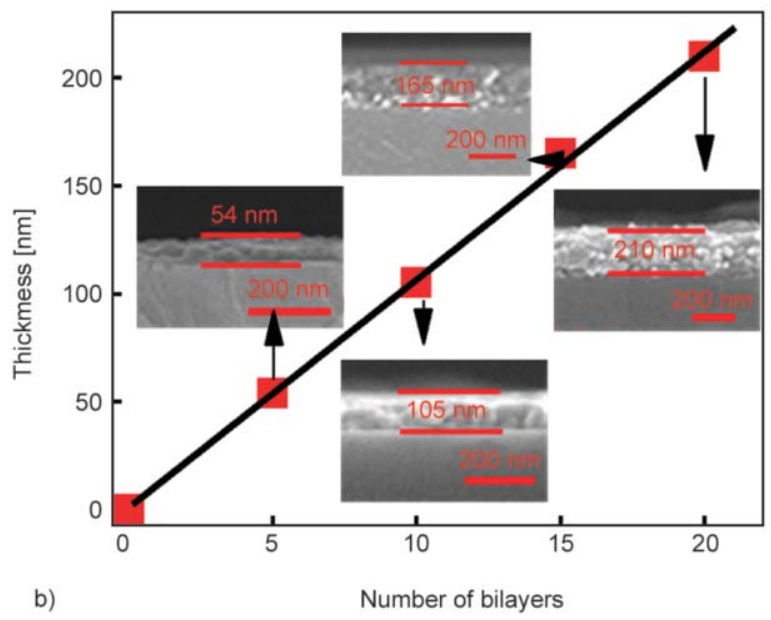

Figure 5. SEM images of PLA/(CNCs/Mg-Al-Eu LDHs) ${ }_{20}$ films: the top-view (a) and the side-view (b). 
and nanofillers. It was believed that the existence of CNCs as nanofillers, presenting a twisted ribbon between PLA and Mg-Al-Eu LDHs, could enhance the nanofiller-polymer interactions [26].

As the thickness of the assembled layers was a critical factor influencing the properties of composite films, the growth of the LBL process was monitored by analyzing the increment in the lateral view of SEM images. According to Figure 5b, the thickness of 5, 10, 15 and 20 bilayers films grown on the glass substrate were 54, 105, 165 and $210 \mathrm{~nm}$, respectively. Aminolyzed quartz worked as the substrate to assemble Mg-Al-Eu LDHs and CNCs. It was worthwhile mentioning that there was a constant increase with the increasing number of bilayers. Based on that, it was estimated the thickness of each LDHs/CNCs bilayer was about $10.75 \pm 0.25 \mathrm{~nm}$. In the meanwhile, the composite films with specific thickness could be prepared by regulating the assembly cycle.

To better understand the LBL assembly process, the UV-vis absorbance spectrum of PLA/(CNCs/Mg-Al$\mathrm{Eu}$ LDHs $)_{\mathrm{n}}$ films was measured. As shown in Figure 6, the UV-vis absorbance of PLA/(CNC/Mg-Al$\mathrm{Eu} \mathrm{LDH})_{\mathrm{n}}$ composite films increased gradually, which was consistent with a typical LBL assembly process [32]. Particularly, the adsorption intensity at $215 \mathrm{~nm}$ rose linearly with the continuous cycle of assembly, which represented a stepwise and regular film growth in thickness. All the results verified the feasibility of preparing a thickness controllable composite membrane.

Mg-Al-Eu LDHs and Mg-Al LDHs fluoresced under selective excitation of 514 and $633 \mathrm{~nm}$, respectively. To evaluate the interactions between CNCs and LDHs nanosheets in the nanocomposites, $\mathrm{Mg}$-Al-Eu LDHs and $\mathrm{Mg}$-Al LDHs were alternately assembled with CNCs. The fluorescence properties of four bilayers composite membranes were characterized, as shown in Figure 7c, the composite membranes emitted bicolor fluorescence under dual wavelength excitation. By alternately assembling with CNCs, the luminescent LDHs particles performed a positioning effect between the layers. Therefore, Mg-Al-Eu LDHs and Mg-Al LDHs fluoresced at specific excitation wavelength without interference, as shown in Figure 7a and $7 \mathrm{~b}$. And the fluorescence intensity gradually rose with the increase of the assembled layers as demonstrated in Figure 7d. It was further indicated that each layer assembled by the LBL process was uniform and orderly, which immobilized the CNCs and LDHs, and thus realized the microscopic controllability of the optical functional molecular spacing [33]. As a result, the luminescence of quenching that may be caused by the accumulation of photoactive molecules was suppressed. As we all know, to obtain the arbitrarily adjustable illuminating color in the visible light range was the goal that researchers were pursuing [34]. Considering the results above, based on

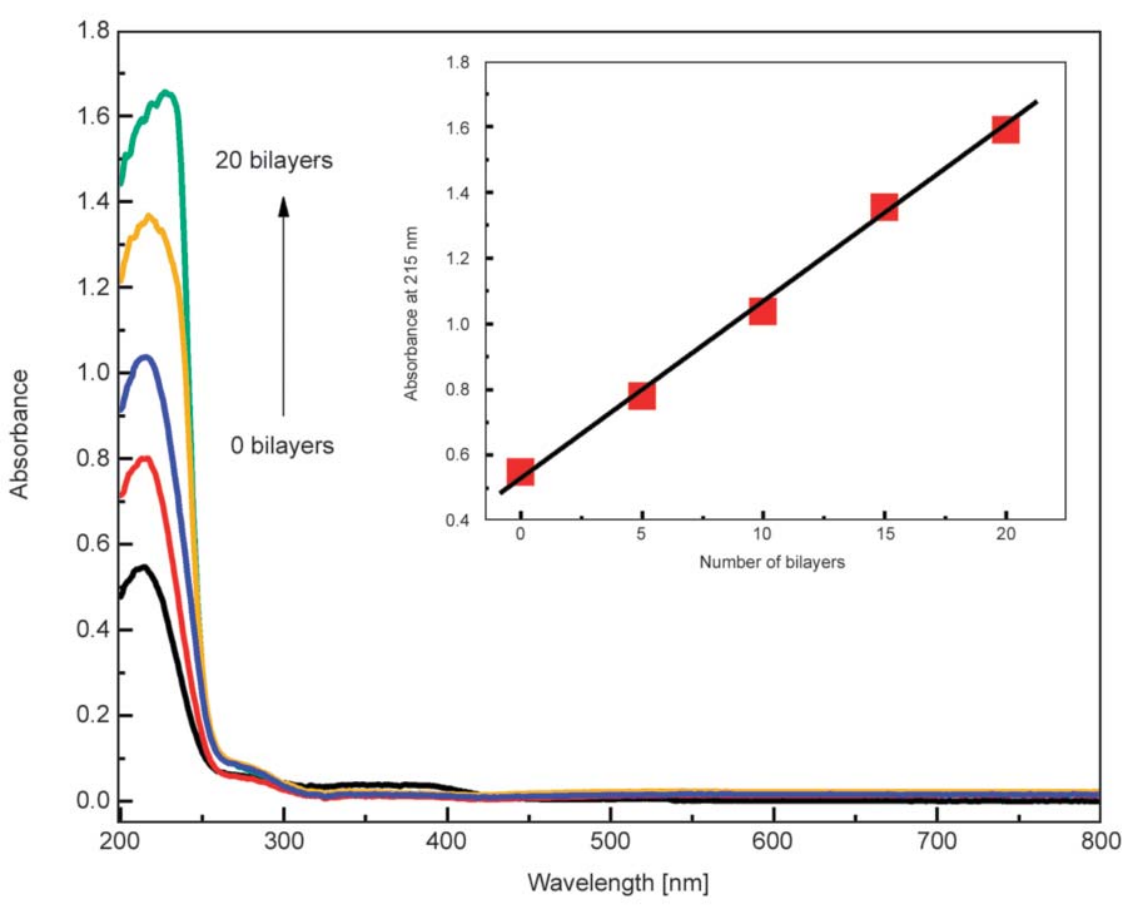

Figure 6. The UV-vis absorption spectrum of PLA/(CNCs/Mg-Al-Eu LDHs $)_{\mathrm{n}}(n=0,5,10,15,20)$ films (The insert shows the dependence of absorbance at $215 \mathrm{~nm}$ as a function of deposition cycles). 


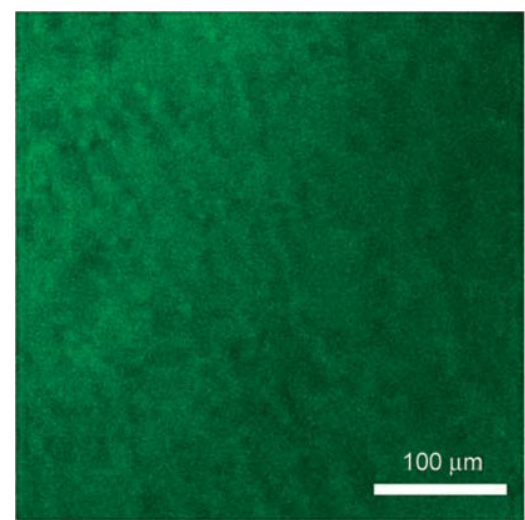

a)

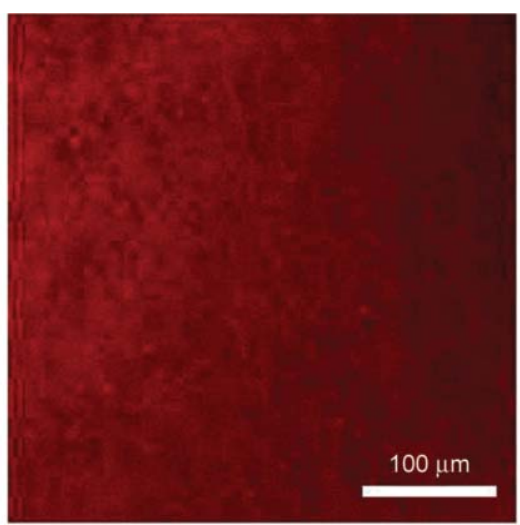

b)

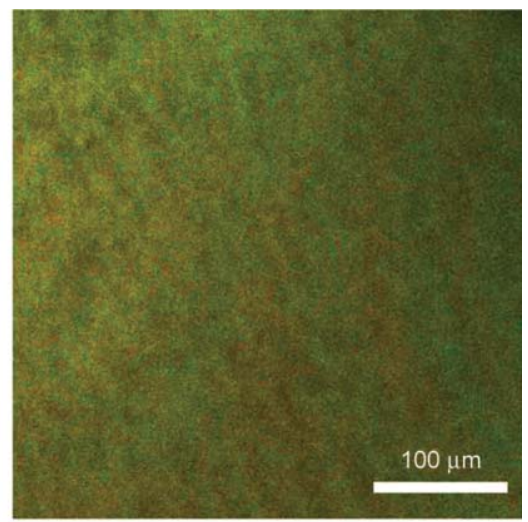

c)

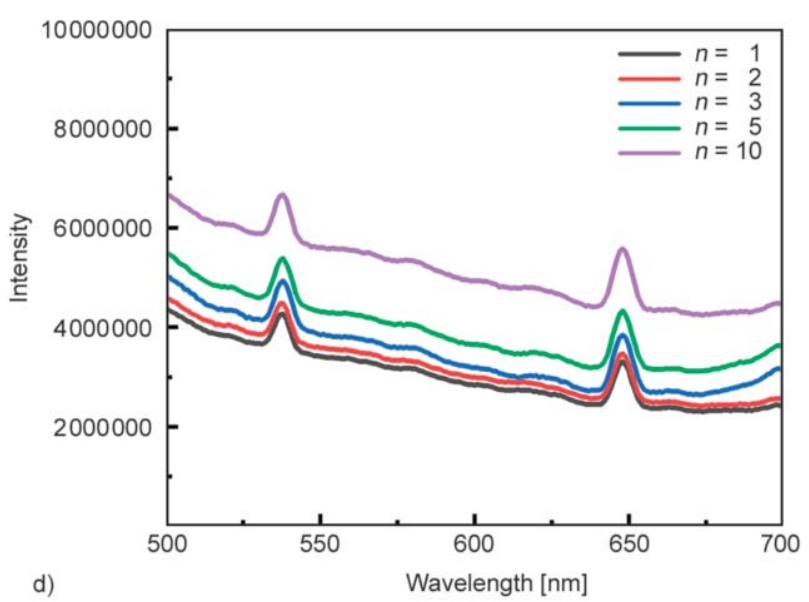

Figure 7. The CLSM image (Fluorescence images under $514 \mathrm{~nm}$ (a), $633 \mathrm{~nm}$ (b) and the 514-633 nm dual wavelength (c) and PLES intensity (d) of the PLA/(CNCs/Mg-Al LDHs) $)_{\mathrm{n}} /(\mathrm{CNCs} / \mathrm{Mg}-\mathrm{Al}-\mathrm{Eu} \mathrm{LDHs})_{\mathrm{n}}$ film.

the diversity of electrostatic assembly forms and the principle of three primary colors, multi-color luminescent composite films were expected to be constructed and used in full color display and optoelectronic devices.

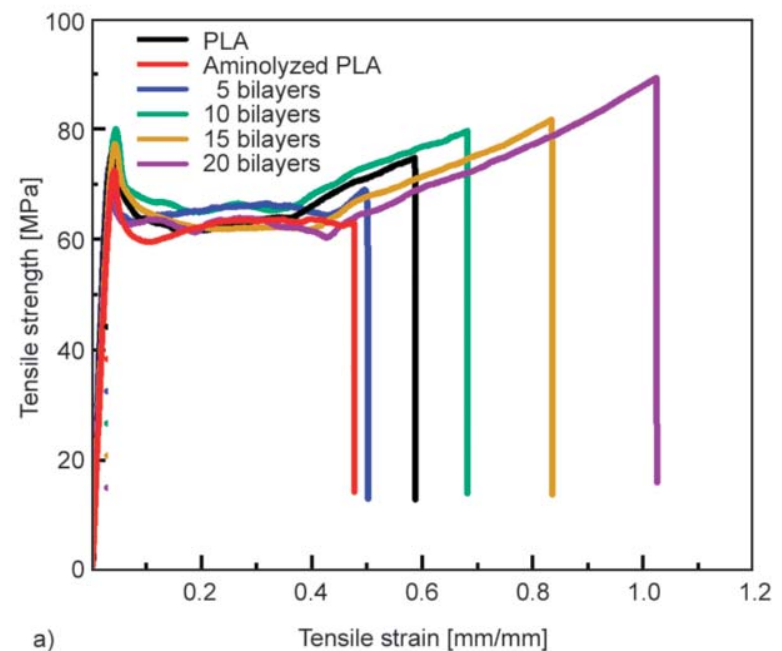

\subsubsection{Properties of composite membrane}

The tensile curves of pure PLA and PLA/(CNCs/ $\mathrm{Mg}-\mathrm{Al}-\mathrm{Eu} \mathrm{LDHs})_{\mathrm{n}}$ films were listed in Figure 8. It was found that the tensile strength of the aminolyzed PLA was lower compared with the original pure PLA.

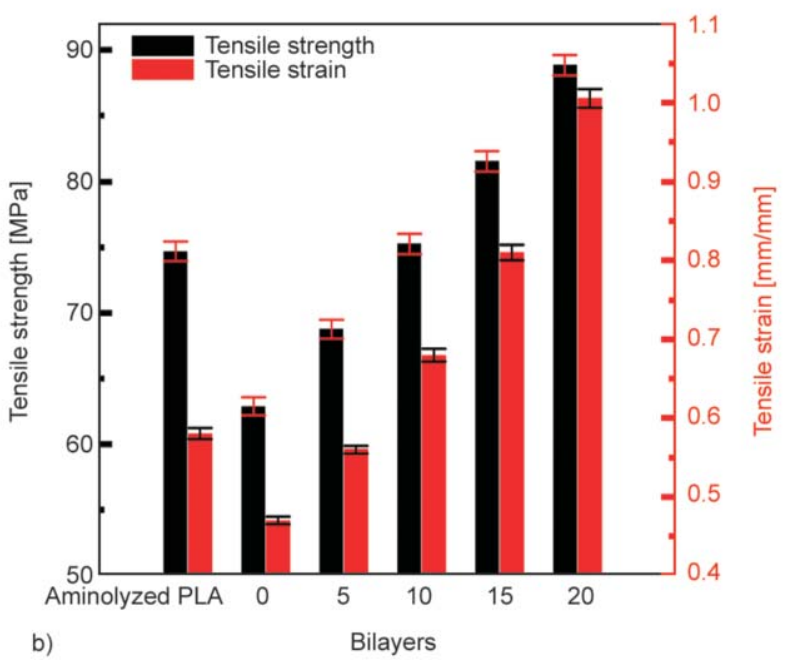

Figure 8. Tensile strain-strength curves and the histograms of the PLA/(CNCs/Mg-Al-Eu LDHs) ${ }_{\mathrm{n}}$ composite membranes. a) Tensile strain-strength curves of PLA/(CNCs/Mg-Al-Eu LDHs $)_{n}$ b) strain and strength histograms of PLA/ $(\mathrm{CNCs} / \mathrm{Mg}-\mathrm{Al}-\mathrm{Eu} \mathrm{LDHs})_{\mathrm{n}}$. 
The decrease of mechanical properties should be attributed to the aminolysis process which destroyed the surface structure of PLA [20,35]. When the CNCs as well as Mg-Al-Eu LDHs alternately distributed on the surface of the PLA film and formed a dense network, the structure greatly enhanced the interaction between the components and brought about an increase in the mechanical properties. When the number of assembled layers was about 10 bilayers, the tensile strength of the composite film was even higher than that of the original pure PLA. It may account for the dense and homogeneous distribution of cellulose nanowhiskers in each layer maximized the interaction between each component and allowed the incorporation of high amounts of the nanofillers [36].

The fluorescence characteristics of the composite membranes were also characterized by CLSM and PLES. As shown in Figure 9a, the composite membrane emitted uniform fluorescence under visible light excitation at $514 \mathrm{~nm}$, further confirming that the LDHs luminophore evenly distributed on the film. As shown in Figure 9b, the fluorescence intensity of the composite film gradually rose with the increasing number of the assembled layers. In virtue of the sharp and intense emission bands of $\mathrm{Eu}^{3+}[37$, 38], the composite films which were merely assembled with Eu-doped LDHs (Figure 9b) showed higher fluorescence performance than that assembled with Mg-Al LDHs and Mg-Al-Eu LDHs (Figure 7d). Besides, the fluorescence spectrum didn't show any red shift or broadening, proving that the interaction

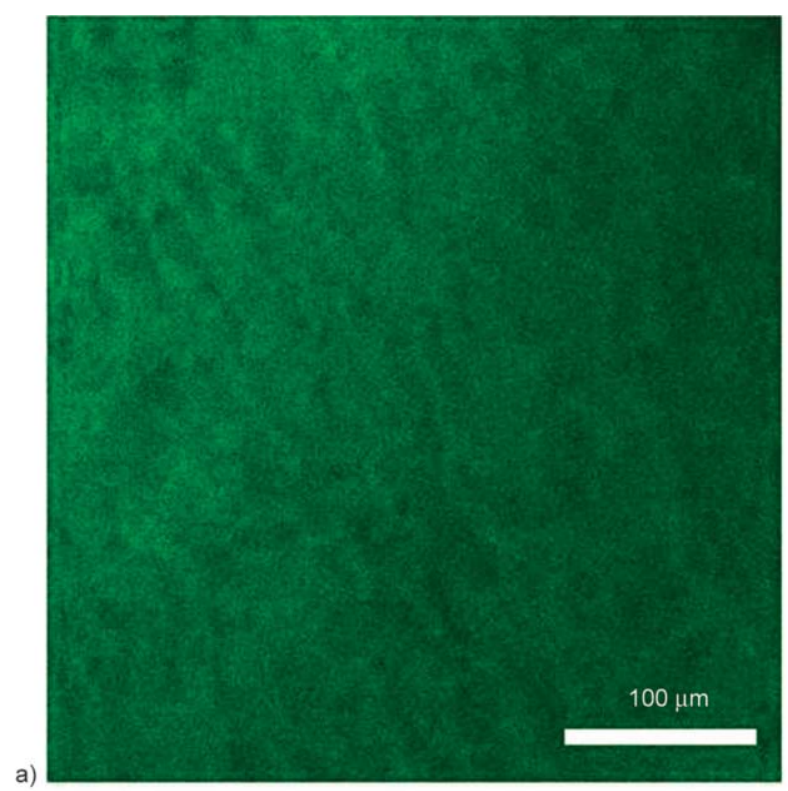

between the molecules and the microenvironment of layers didn't vary significantly during the assembly process, and it was consistent with the reference [39]. From the mounting fluorescence intensity in combination with the comparatively smooth surface and the uniform increment of thickness in Figure 5b, it was indicated that the flaky LDHs nanosheets were stacked in a parallel way on the surface of PLA.

Fluorescence stability was a vital parameter to comprehend the excited state information of fluorescent molecules. Since all fluorophores were photobleached upon continuous illumination, especially in fluorescence microscopy where the light intensities were high, the fluorescence stability of the composite membrane was detected under $514 \mathrm{~nm}$ by means of a highintensity laser of CLSM [40]. As indicated in Figure 10, the fluorescence intensity of the composite film didn't decline to $96 \%$ until the bleaching repeated over 1000 times about $1000 \mathrm{~s}$, showing a superior bleachability compared with the literature which decreased $10 \%$ after continuous excitation for $10 \mathrm{~min}$ [41]. There was a relative obvious decline in fluorescence intensity of $\mathrm{PLA} /(\mathrm{CNCs} / \mathrm{Mg}-\mathrm{Al}-\mathrm{Eu} \mathrm{LDHs})_{10}$ after bleaching over 1000 times, remaining around $70 \%$ of that of the unbleached film after bleaching 6000 times. In brief, the composite membranes possessed a stable fluorescence performance.

\section{Conclusions}

CNCs and LDHs nanosheets presenting negative and positive surface variations had been successfully

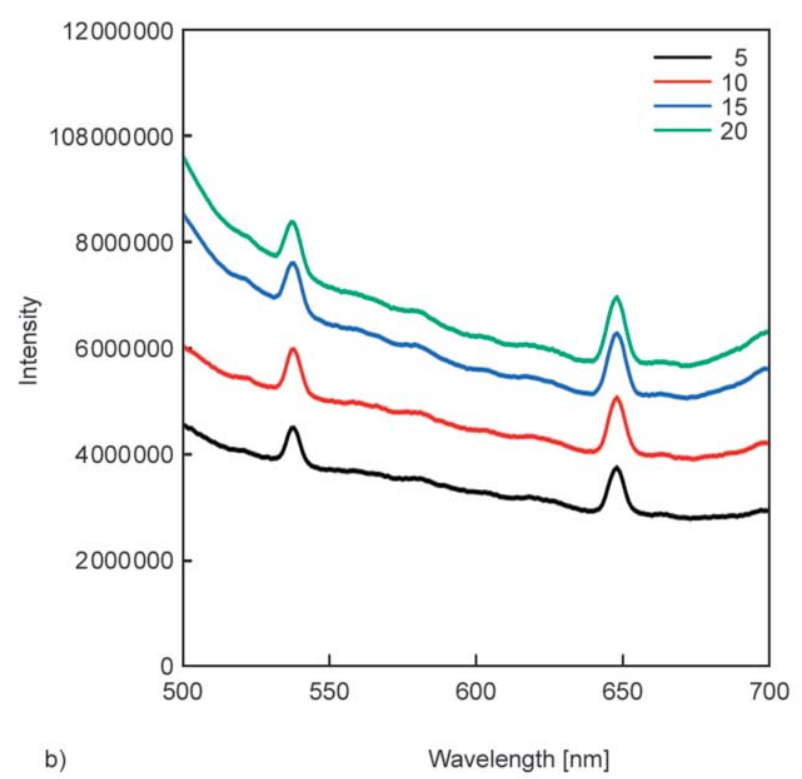

Figure 9. The CLSM image (under $514 \mathrm{~nm}$ ) of PLA/(CNCs/Mg-Al-Eu LDHs) 10 (a) and PLES intensity of the $\mathrm{PLA} /(\mathrm{CNCs} / \mathrm{Mg}-\mathrm{Al}-\mathrm{Eu} \mathrm{LDHs})_{\mathrm{n}}$ composite membrane (b). 


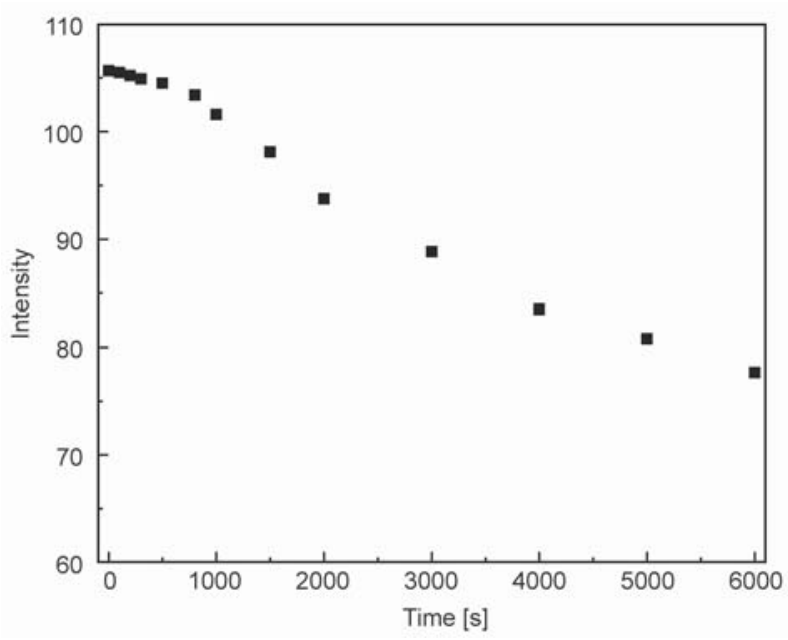

Figure 10. Fluorescence intensity (under $514 \mathrm{~nm}$ ) changes of the PLA/(CNCs/Mg-Al-Eu LDHs) composite membrane.

deposited onto aminolyzed PLA membranes through electrostatic layer-by-layer assembly. The consecutive increase of CNCs and Mg-Al-Eu LDHs nanosheets was monitored by SEM images, UV-vis spectroscopy and fluorescence emission. More specifically, the SEM image characterization showed that the amount of deposited materials was similar for each adsorbed layer with an average thickness of $10.8 \pm 0.2 \mathrm{~nm}$ for each bilayer of composite films. The CLSM and PLES presented that the fluorescence properties increased with the incremental number of bilayers, indicating the establishment of the fluorescent network. After being repeatedly bleached 1000 and 6000 times by CLSM, the fluorescence intensity of the PLA/(CNCs/Mg-Al-Eu LDHs $)_{10}$ film still maintained above 96 and $70 \%$ of the unbleached, respectively. It displayed satisfactory optical stabilities and fluorescence stability, unlike that of conventional fluorescent materials. Based on above experimental data and results, it was expected that the size-controlled multilayer fluorescent materials were potentially used in the application of anti-counterfeiting packaging, color display or optoelectronic devices.

\section{Acknowledgements}

The authors acknowledge the Natural Science Foundation of China (No. 31670586), Science and Technology Planning Project of Guangdong Province (2016A020221011), the Fundamental Research Funds for the Central Universities (2017MS073).

\section{References}

[1] Madhavan Nampoothiri K., Nair N. R., John R. P.: An overview of the recent developments in polylactide (PLA) research. Bioresource Technology, 101, 84938501 (2010).

https://doi.org/10.1016/j.biortech.2010.05.092

[2] Amor A., Okhay N., Guinault A., Miquelard-Garnier G., Sollogoub C., Gervais M.: Combined compatibilization and plasticization effect of low molecular weight poly (lactic acid) in poly(lactic acid)/poly(3-hydroxybutyrate-co-3-hydroxyvalerate) blends. Express Polymer Letters, 12, 114-125 (2018).

https://doi.org/10.3144/expresspolymlett.2018.10

[3] Chow W. S., Teoh E. L., Karger-Kocsis J.: Flame retarded poly(lactic acid): A review. Express Polymer Letters, 12, 396-417 (2018). https://doi.org/10.3144/expresspolymlett.2018.34

[4] van Tassel P. R.: Polyelectrolyte adsorption and layerby-layer assembly: Electrochemical control. Current Opinion in Colloid and Interface Science, 17, 106-113 (2012). https://doi.org/10.1016/j.cocis.2011.08.008

[5] Halász K., Hosakun Y., Csóka L.: Reducing water vapor permeability of poly(lactic acid) film and bottle through layer-by-layer deposition of green-processed cellulose nanocrystals and chitosan. International Journal of Polymer Science, 2015, 954290/1-954290/6 (2015).

https://doi.org/10.1155/2015/954290

[6] Frone A. N., Panaitescu D. M., Chiulan I., Nicolae C. A., Vuluga Z., Vitelaru C., Damian C. M.: The effect of cellulose nanofibers on the crystallinity and nanostructure of poly(lactic acid) composites. Journal of Materials Science, 51, 9771-9791 (2016). https://doi.org/10.1007/s10853-016-0212-1

[7] Hussain S., Dickson A. R.: Improving and predicting the mechanical properties of foamed and stretched composite poly(lactic acid) films. Express Polymer Letters, 13, 18-26 (2019). https://doi.org/10.3144/expresspolymlett.2019.3

[8] Montes S., Etxeberria A., Mocholi V., Rekondo A., Grande H., Labidi J.: Effect of combining cellulose nanocrystals and graphene nanoplatelets on the properties of poly(lactic acid) based films. Express Polymer Letters, 12, 543-555 (2018). https://doi.org/10.3144/expresspolymlett.2018.45

[9] Mochane M. J., Mokhena T. C., Mokhothu T. H., Mtibe A., Sadiku E. R., Ray S. S., Ibrahim I. D., Daramola O. O.: Recent progress on natural fiber hybrid composites for advanced applications: A review. Express Polymer Letters, 13, 159-198 (2019). https://doi.org/10.3144/expresspolymlett.2019.15

[10] Zhang Z., Chen G., Liu J.: Tunable photoluminescence of europium-doped layered double hydroxides intercalated by coumarin-3-carboxylate. RSC Advances, 4, 7991-7997 (2014).

https://doi.org/10.1039/c3ra46930a 
[11] Liu M., Wang T., Ma H., Yu F., Hu K., Guan C.: Assembly of luminescent ordered multilayer thin-films based on oppositely-charged MMT and magnetic NiFeLDHs nanosheets with ultra-long lifetimes. Scientific Reports, 4, 7147/1-7147/9 (2014). https://doi.org/10.1038/srep07147

[12] Liu M., Wang T., Ma H., Fu Y., Hu K., Guan C.: Layerby-layer assembly of luminescent multilayer thin films by MMT, anionic chromophores and magnetic CoAlLDHs nanosheets. Materials Letters, 153, 40-43 (2015). https://doi.org/10.1016/j.matlet.2015.04.002

[13] Tanaka T., Nishimoto S., Kameshima Y., Miyake M.: Fabrication of layered double hydroxide/photoresponsive dendron nanocomposite multilayer film by electrostatic layer-by-layer assembly. Materials Letters, 65, 2315-2318 (2011). https://doi.org/10.1016/j.matlet.2011.05.004

[14] Yan D., Lu J., Wei M., Evans D. G., Duan X.: Recent advances in photofunctional guest/layered double hydroxide host composite systems and their applications: Experimental and theoretical perspectives. Journal of Materials Chemistry, 21, 13128-13139 (2011). https://doi.org/10.1039/C1JM11594D

[15] Ma H., Gao R., Yan D., Zhao J., Wei M.: Organic-inorganic hybrid fluorescent ultrathin films and their sensor application for nitroaromatic explosives. Journal of Materials Chemistry C, 1, 4128-4137 (2013).

https://doi.org/10.1039/c3tc30142g

[16] Li C., Wei M., Evans D. G., Duan X.: Recent advances for layered double hydroxides (LDHs) materials as catalysts applied in green aqueous media. Catalysis Today, 247, 163-169 (2015).

https://doi.org/10.1016/j.cattod.2014.05.032

[17] Leroux F., Besse J-P.: Polymer interleaved layered double hydroxide: A new emerging class of nanocomposites. Chemistry of Materials, 13, 3507-3515 (2001). https://doi.org/10.1021/cm0110268

[18] Chen H., Hu L., Chen M., Yan Y., Wu L.: Nickel-cobalt layered double hydroxide nanosheets for high-performance supercapacitor electrode materials. Advanced Functional Materials, 24, 934-942 (2014).

https://doi.org/10.1002/adfm.201301747

[19] Coiai S., Cicogna F., de Santi A., Pérez Amaro L., Spiniello R., Signori F., Fiori S., Oberhauser W., Passaglia E.: MMT and LDH organo-modification with surfactants tailored for PLA nanocomposites. Express Polymer Letters, 11, 163-175 (2017).

https://doi.org/10.3144/expresspolymlett.2017.18

[20] Gong Y., Zhu Y., Liu Y., Ma Z., Gao C., Shen J.: Layerby-layer assembly of chondroitin sulfate and collagen on aminolyzed poly(L-lactic acid) porous scaffolds to enhance their chondrogenesis. Acta Biomaterialia, 3, 677-685 (2007).

https://doi.org/10.1016/j.actbio.2007.04.007
[21] Shahabi-Ghahafarrokhi I., Khodaiyan F., Mousavi M., Yousefi H.: Preparation and characterization of nanocellulose from beer industrial residues using acid hydrolysis/ultrasound. Fibers and Polymers, 16, 529-536 (2015).

https://doi.org/10.1007/s12221-015-0529-4

[22] Liu D. Y., Yuan X. W., Bhattacharyya D., Easteal A. J.: Characterisation of solution cast cellulose nanofibre reinforced poly(lactic acid). Express Polymer Letters, 4, 26-31 (2010). https://doi.org/10.3144/expresspolymlett.2010.5

[23] Duan X., Li F.: Applications of layered double hydroxides. in 'Layered double hydroxides' (eds.: Duan X., Evans D. G.) Springer, Berlin, 193-224 (2006). https://doi.org/10.1007/430_007

[24] Prathapan R., Thapa R., Garnier G., Tabor R. F.: Modulating the zeta potential of cellulose nanocrystals using salts and surfactants. Colloids and Surfaces A: Physicochemical and Engineering Aspects, 509, 11-18 (2016). https://doi.org/10.1016/j.colsurfa.2016.08.075

[25] Choi K., Seo S., Kwon H., Kim D., Park Y. T.: Fire protection behavior of layer-by-layer assembled starchclay multilayers on cotton fabric. Journal of Materials Science, 53, 11433-11443 (2018).

https://doi.org/10.1007/s10853-018-2434-x

[26] Trigueiro J. P., Silva G. G., Pereira F. V., Lavall R. L.: Layer-by-layer assembled films of multi-walled carbon nanotubes with chitosan and cellulose nanocrystals. Journal of Colloid and Interface Science, 432, 214-220 (2014).

https://doi.org/10.1016/j.jcis.2014.07.001

[27] de Mesquita J. P., Donnici C. L., Pereira F. V.: Biobased nanocomposites from layer-by-layer assembly of cellulose nanowhiskers with chitosan. Biomacromolecules, 11, 473-480 (2010).

https://doi.org/10.1021/bm9011985

[28] Sun H., Chu Z., Hong D. H., Zhang G., Xie Y., Li L., Shi K.: Three-dimensional hierarchical flower-like MgAl-layered double hydroxides: Fabrication, characterization and enhanced sensing properties to $\mathrm{NO}_{\mathrm{x}}$ at room temperature. Journal of Alloys and Compounds, 658, 561-568 (2016).

https://doi.org/10.1016/j.jallcom.2015.10.237

[29] Prinetto F., Ghiotti G., Graffin P., Tichit D.: Synthesis and characterization of sol-gel $\mathrm{Mg} / \mathrm{Al}$ and Ni/Al layered double hydroxides and comparison with co-precipitated samples. Microporous and Mesoporous Materials, 39, 229-247 (2000). https://doi.org/10.1016/s1387-1811(00)00197-9

[30] Zhang W., Li Y., Fan H.: Layer-by-layer assembly of luminescent ultrathin films by $\mathrm{Mg}-\mathrm{Al}-\mathrm{Eu} \mathrm{LDHs}$ nanosheets and organic ligand with high transparency. Optical Materials, 51, 78-83 (2016).

https://doi.org/10.1016/j.optmat.2015.11.001 
[31] Chen D., Wang X., Liu T., Wang X., Li J.: Electrically conductive poly(vinyl alcohol) hybrid films containing graphene and layered double hydroxide fabricated via layer-by-layer self-assembly. ACS Applied Materials and Interfaces, 2, 2005-2011 (2010).

https://doi.org/10.1021/am100307v

[32] Celebi H., Kurt A.: Effects of processing on the properties of chitosan/cellulose nanocrystal films. Carbohydrate Polymers, 133, 284-293 (2015). https://doi.org/10.1016/j.carbpol.2015.07.007

[33] Yan D., Lu J., Ma J., Wei M., Evans D. G., Xue D.: Fabrication of an anionic polythiophene/layered double hydroxide ultrathin film showing red luminescence and reversible $\mathrm{pH}$ photoresponse. AIChE Journal, 57, 19261935 (2011).

https://doi.org/10.1002/aic.12400

[34] Yan D., Lu J., Wei M., Qin S., Chen L., Zhang S., Evans D. G., Duan X.: Heterogeneous transparent ultrathin films with tunable-color luminescence based on the assembly of photoactive organic molecules and layered double hydroxides. Advanced Functional Materials, 21, 2497-2505 (2011).

https://doi.org/10.1002/adfm.201002446

[35] Zhu Y., Gao C., Liu X., Shen J.: Surface modification of polycaprolactone membrane via aminolysis and biomacromolecule immobilization for promoting cytocompatibility of human endothelial cells. Biomacromolecules, 3, 1312-1319 (2002).

https://doi.org/10.1021/bm020074y

[36] Podsiadlo P., Choi S-Y., Shim B., Lee J., Cuddihy M., Kotov N. A.: Molecularly engineered nanocomposites: Layer-by-layer assembly of cellulose nanocrystals. Biomacromolecules, 6, 2914-2918 (2005).

https://doi.org/10.1021/bm050333u
[37] Chang Z., Evans D., Duan X., Boutinaud P., de Roy M., Forano C.: Preparation and characterization of rare earth-containing layered double hydroxides. Journal of Physics and Chemistry of Solids, 67, 1054-1057 (2006). https://doi.org/10.1016/j.jpcs.2006.01.025

[38] Yan D., Lu J., Wei M., Han J., Ma J., Li F., Evans D. G., Duan X.: Ordered poly( $p$-phenylene)/layered double hydroxide ultrathin films with blue luminescence by layer-by-layer assembly. Angewandte Chemie International Edition, 48, 3073-3076 (2010). https://doi.org/10.1002/anie.200900178

[39] Shiragami T., Mori Y., Matsumoto J., Takagi S., Inoue H., Yasuda M.: Non-aggregated adsorption of cationic metalloporphyrin dyes onto nano-clay sheets films. Colloids and Surfaces A: Physicochemical and Engineering Aspects, 284, 284-289 (2006). https://doi.org/10.1016/j.colsurfa.2005.11.102

[40] Wolf E., Schrüber A.: Phycobiliprotein fluorescence of Nostoc punctiforme changes during the life cycle and chromatic adaptation: Characterization by spectral confocal laser scanning microscopy and spectral unmixing. Plant, Cell and Environment, 28, 480-491 (2005). https://doi.org/10.1111/j.1365-3040.2005.01290.x

[41] Li X., Xie Y., Song B., Zhang H-L., Chen H., Cai H., Liu W., Tang Y.: A stimuli-responsive smart lanthanide nanocomposite for multidimensional optical recording and encryption. Angewandte Chemie, 56, 2689-2693 (2017).

https://doi.org/10.1002/anie.201700011 